\title{
ON SULPHAEMOGLOBINAEMIA
}

\author{
By W. C. LONG AND E. I. SPRIGGS
}

(From the Duff House Laboratories)

IN vol. vii of this Journal Dr. R. L. Mackenzie Walls gave a brief account of the cases of sulphaemoglobinaemia hitherto recorded. $\mathrm{He}$ also reviewed the literature of the disease, which was first described by van den Bergh, and communicated the results of his own researches. At that time there were eleven authentic examples. The condition has probably been recognized more than once since then, but is rare enough to justify a detailed description of another case.

An unmarried lady, aged 32, was admitted on September 14, 1916. The complexion was pale, the lips blue.

She stated that she was always 'breaking down', and complained of constipation, nausea, frequent vomiting, a constant ache below the right costal margin, pain in the lower part of the abdomen, also in the head, back, and limbs; and of weakness and fatigue.

Her parents were living and healthy. Two brothers were alive, one the subject of chronic phthisis.

The medical history, as related by the patient, was as follows: When a few months old she had an abscess in the brain, from which she nearly died. She does not think there were any fits or convulsions. Adenoids and tonsils were removed in early life. She had always been nervous and considered peculiar. At school she had attacks of vomiting and pain in the abdomen. These would last as long as a month. Such an attack was at least once brought on by a fright. In the intervals she was well and able to take ordinary food, but always constipated. At this time she was stout.

At 17 she had a sudden attack of severe pain in the left lower abdomen, and was in bed for a month. The left kidney was explored and drained, but no sufficient lesion found to explain the symptoms. A long period of ill health followed, spent in nursing homes, and she became thin. The attacks of vomiting ceased, but she suffered from a severe 'chill', causing fever and diarrhoea with blood in the stools. This was diagnosed as colitis and treated with intestinal douches.

From the age of 15 to 25 she was sometimes told that she looked intoxicated, and at these times, though there was no unconsciousness, or physical or mental discomfort, she lost grasp of what was happening for an hour or two or longer. There was no personal grief or worry to account for these feelings.

At 25 the appendix and a band of adhesions were removed. Later, repeated attacks of severe pain oceurred in the right flank, frequently causing vomiting.

[Q. J. M., Jan., r918.] 
At 27 right laparotomy was performed and adhesions removed, after which no further pain was felt in that region; but she was often laid up in bed and in charge of nurses, suffering from frequent faintness, anaemia, sleeplessness, and persistent constipation, but without scvere pain.

In July 1915 (aged 31) she had cystitis, since when there had been irritation of the bladder. In the latter part of the year she was helping in an infirmary, though in poor health, becoming easily fatigued and inclined to be hysterical. One night she suddenly felt faint, suffocated, and numb, and her colour became blue; she did not lose consciousness and knew what was going on around her. 'This was the first attack of cyanosis of which she is aware. The blueness persisted and soon after she had a similar sudden attack, which lasted for some hours and left her very weak. Constipation was still troublesome.

In July 1916 severe pain was felt below the right costal margin, passing through to the back. No fever, vomiting, or rigor occurred, though she frequently felt faint. This pain persisted, though less severely, until she came to Duff House.

Menstruation began at 14 years of age and was usually irregular. A year ago a severe haemorrhage occurred and caused faintness. For the past six months there had been amenorrhoea. For some months she had occasionally a brown offensive vaginal discharge. This was not complained of during her stay.

Symptoms on admission. Constipation. She had always taken aperients since childhood. A motion was usually preceded by colicky pain.

Vomiting. Frequent, and commonly without nausea. The act of stooping down after an ordinary meal, sudden exciternent, or fright, or certain foods, especially cream and milk, might bring it on.

The appetite was capricious.

Pain. A constant ache below the right costal margin, sometimes sharp, severe, and extending through to the back. It had no particular relation to food or occupation, was accompanied by nausea and sometimes by sickness. There was also general discomfort in other parts of the abdomen, 'a gnawing, aching sensation,' especially in tho hypogastrium. A severe ache was frequently complained of in the sacral region, usually worse at night and increased at the periods. Other pains were complained of in various parts of the body and limbs, and neuralgic pains in the right side of the head and neek, partieularly affecting the light ear: these were sometimes relieved by aspirin.

Palpitation would occur without excitement or exertion.

The patient felt tired and 'not sure of herself'.

Examination. The complexion was pale, dull, muddy, and dark round the eyes, which were a little prominent. The lips were bluish, the features tired and expressionless. The skin was dry, though there was sometimes profuse perspiration at night. T'he forearms showed slight subcutaneous swelling which did not pit on pressure. There were mild bruises on the arms and legs: the patient said that she bruised easily. The manner was composed and agreeable, though cerebration was rather slow: she was vague in giving an account of herself, but spoke of pain and discomfort in superlatives. The temperature was normal.

The tongue was clean. The teeth were partly artificial, deficient in molars, and carious; the gums were pale and firm. The pharynx was pale, dry, and cicatrized. 'The thyroid was natural. No enlarged glands were felt. The abdomen was flat and its walls thin. On the least pretence of an examination being made the muscles were rigidly contracted, making satisfactory palpation impossible. Considerable pressure revealed tenderness below the right costal margin. The area of splenic dullness was enlarged, extending from the sixth rib to the costal margin. The spleen could not be felt.

The cardiac impulse was in the fourth intercostal space. A faint systolic 
bruit accompanied the first sound at the mitral area. The pulse was regular, of poor volume; the blood pressure $135 \mathrm{~mm}$. $\mathrm{Hg}$.

The blood contained 65 per cent. haemoglobin, 2,350,000 red cells, and 5,400 white cells in the cubic nillimetre. No abnormal types of cells were found. A differential blood-count of 500 cells gave :

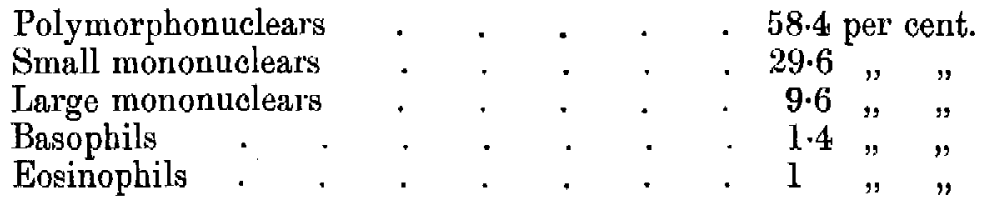

Blood cultures were sterile.

The knee-jerks were absent, but other nervous reflexes were normal.

An X-ray examination after a barium meal showed that the stomach was long, contracted to a tubular shape, and emptied rapidly. The bulk of the meal loft the stomach in twenty minutes, though there was a small residue for four hours. There was delay at one point in the jejunal coils. Ileal stasis was present, material being still contained in the terminal ileum six hours after the stomach was empty. There was great delay throughout the colon, especially in the proximal portion, the bulk of the meal lying in the transverse colon and only a small portion having reached the pelvic colon at seventy-two hours.

A twenty-four hours' collection of urine, taken on admission, measured 920 c.c. and contained diacetic acid and acetone.

The stools were reddish-brown, partially fluid, offensive, and contained much undigested vegetable matter and varying amounts of mucus.

A provisional diagnosis was made of severe anaemia with cyanosis of unknown cause.

Progress. On the third day after admission the patient began to vomit all food taken. The vomiting was seldom preceded by nausea or other discomfort. Solid and liquid food as well as medicine were bronght up without effort a few minutes after being swallowed. 'The vomiting continued for about two weeks. Nutrition suffered very little and the general physical condition remained unaltered. During this period the urine was diminished to 200 to 400 c.c. in twenty-four hours, the total nitrogen varying from $1.7 \mathrm{grm}$. to $3.6 \mathrm{grm}$. Diacetic acid and acetone disappeared from the urine the seventh day after admission and did not reappear.

Pain and discomfort in the bladder were frequently complained of and could not be explained. The quantity of urine became further diminished, the total collection in twenty-four hours varying during October from hardly any to 250 c.c., the average being 107 c.c. As at this time the total nitrogen was only 0.7 to $2.0 \mathrm{grm}$, per diem, whilst that in the food retained was about $5 \mathrm{grm}$, per day, and diacetic acid and acetone were absent, we could not avoid the suspicion that all the urine did not reach the laboratory; but though precautions were taken, this was not proved.

The abdominal pains varied in site and character, the most constant and severe being below the right costal margin. The patient lay on her back or side with the right thigh flexed on the abdomen and the knee drawn up to the chest. If questioned, she would say the pain was very severe, but otherwise did not complain or appear to be in great distress. The temperature and pulse were normal; no rigors or sweats occurred. Various measures to relieve this pain were tried unsuccessfully. Morphia was given hypodermically on three or four occasions, but secmed to excite without giving relief. Insomnia was persistent.

Early in October, while lying on a couch in the open air, the patient became dusky in colour, looked very vacant and strange, and appeared to have fainted. The nurse who was summoned and had her removed to bed was alarmed and thought she had had a 'heart attack'. She quickly became more blue, and was 
unconscious for a short time. When seen later she was conscious but exhausted, and could give no account of her attack. Cyanosis was obvious, and, though gradually lessening, it did not entirely disappear before the second attack, which. occurred a few days later.

Second attack, October 16. The patient had felt poorly all day, but had been unusually free from pain. She took no meal in the evening, but was sitting up and talking to friends in her room. At 8 p.m. she looked strange, had a vacant stare, appeared to be fainting or going into a swoon, and then rapidly became unconscious. At 8.45 p.m. - that is, three-quarters of an hour after the onset-she was lying flat on her back in bed. Her appearance gave the impression of a dying person. The skin was of a dusky leaden colour, an extreme pallor, modified by the biuish tint. The lips, nostrils, and mucous membrane of the mouth were a dull deep purple. The extrenities were a lavender-blue colour, best seen in the nails. She was apparently unconscious. The eyelids were half open and the eyes staring fixedly to the right. The corneal reflex was slow and feeble. 'The pupils were dilated, but reacted to a bright light stimulus. The body' and limbs were warm, partly due to the hot bottles and blankets provided. The limbs were flaccid, and there was no rigidity of any of the muscles. The abdomen conld easily be palpated and the opportunity, not previously afforded, was taken to examine it carefully. Nothing abnormal could be felt.

The temperature was $97^{\circ} \mathrm{F}$. in the axilla. The pulse was 90 , regular, a little smaller than usual, but of fair volume. The heart sounds were regular. A soft systolic bruit accompanied the first sound, and could be detected all over the cardiac area, but was best heard at the apex. No bruit was heard in the vessels of the neck. Respiration was shallow, 18 to the minute. A deep sighing inspiration occurred at intervals of about forty-five seconds. There was no peculiarity in the odour of the breath. In the course of two hours she slowly became semi-conscious. The tongue moved to moisten the lips and the eyes were slowly moved from side to side. A teaspoonful of brandy was rejected, but sips of water were swallowed. Two pints of saline solution wero given per roctum. and retained for an hour and a half.

At 11.45 p.m. the pulse was 102, regular, and of fair volume. A draught containing liq. strych. $\mathfrak{a}) 3$, tr. digit. a) 5 , and aq. camph. 31 was given and retained. The condition remained the same, but she could be roused, and moved her head in response to inquiries. She seemed to have pain, probably in the abdomen. At 1.20 a.m. the pulse was 88 , and improved in character. Respiration was more natural. She was then conscious and able to speak, complained of numbness all over the body, but had no pain. The strychnine draught was continued two-hourly.

In the morning she had more or less regained her usual appearance, though cyanosis was still deep and she was a good deal exhausted. During the next few days the blueness gradually lessened, but did not disappear.

A third attack took place on October 22, lasting for a little over three hours, during most of which she appeared to be unconscious. The patient had two other attacks during her stay, in all five in seven weeks. Unconsciousness appeared to be complete so far as pricking with a pin and other signs indicated.

Annoyance or disappointment was liable to be followed by an attack.

As there were no cardiac or respiratory symptorns to explain the attacks, nor any signs of thoracic disease, a diagnosis of enterogenous cyanosis as described by Dr. Garrod in Allbutt and Rolleston's System of Medicine was made. The term includes cases of methaemoglobinaemia, due usually, though not always, to drugs, and sulphaemoglobinaemia, so far of undetermined causation. The blood was then examined with the spectroscope and found to contain sulphaemoglobin. 


\section{Spectroscopic Examination of the Blood.}

The blood drawn for examination was always dark and frequently of a deep chocolate colour. On November 2, blood was taken, defibrinated, and examined in various dilutions, the most satisfactory being 1 in 20 and 1 in 30 . Similar dilutions of normal blood were used for control. An absorption band was seen to the left of $D$ in the red. It coincided with the band of artificially prepared sulphaemoglobin when compared by means of a superimposed spectrum. When compared with the spectrum of artificial methaemoglobin, the band of the patient's blood was nearer to the right or violet end. The addition of ammonium sulphide solution caused no alteration in the appearance of the band in the patient's blood, whilst that of methaemoglobin disappeared. Acid-free carbon monoxide, passed through the patient's blood, caused the band to move to the right. The measurements of the band are shown in the accompanying table, with the corresponding measurements given by Wood Clarke and Mackenzie Wallis, as quoted by the latter.

$\begin{array}{lccc} & \begin{array}{c}\text { Patient's } \\ \text { blood. }\end{array} & \begin{array}{c}\text { Wood } \\ \text { Clarke. }\end{array} & \begin{array}{c}\text { Mackenzie } \\ \text { Wallis. }\end{array} \\ \text { Band of sulphaemoglobin } & \cdot 615-31 & 613-29 & 613-25 \\ \text { On addition of CO } & 609-28 & 605-20 & \\ \text { Artificial methaemoglobin } & \text {. } 621-44 & 620-45 & \end{array}$

At a second examination (December 10), when cyanosis was less pronounced, the sulphaemoglobin band was again distinct, but on dilution it disappeared sooner than on the previous occasion. The band could be seen in the lobe of the ear with an ordinary electric lamp and a pocket spectroscope.

By this time (November) the general health had suffered a good deal, and the patient was thinner and weaker. More or less cyanosis was usually present. Nourishment was taken with difficulty and vomiting occurred occasionally. Discomfort in the abdomen was constant and sometimes severe pain was complained of and lack of sleep.

Early in November another X-ray examination was made after $a$ barium meal. The stomach was constricted and presented an hour-glass appearance. The constriction rapidly relaxed on pressure, but with a strong tendency to contract again. 'The stomach emptied in eight hours. The jejunal delay previously seen was less noticeable, but some coils appeared to be bunched and partly fixed in the right subcostal area, corresponding to the site of pain usually felt. There was still moderate ileal stasis, and general delay in the colon, with irregular segmentation in the latter.

The temperature was normal throughout with the exception of a slight rise for forty-eight hours, for which no cause was discovered.

The vomits usually consisted of freshly ingested food and frequently contained no free hydrochloric acid.

On October 22 a blood examination showed $\mathrm{Hb}$. 60, red cells 3,200,000, white cells 7,400 ; the $\mathrm{Hb}$. being slightly less and the red cells rather more than on admission.

\section{Further Observations on Blood, Urine, and Saliva.}

In the earlier cases nitrites were found in the urine. The observation must be made on fresh acid urine, for van den Bergh and Gutterink showed that any urine which has begun to ferment, or any fluid which has stood in air, may contain nitrites. As nitrites can form methaemoglobin from oxyhaemoglobin, and urine, with or without nitrites, can slowly effect the same change, attention has been paid to the urine and to nitrites in sulphaemoglobinaemia also. 
In the cases observed by Dr. Mackenzie Wallis, nitrites were found frequently in the urine and in the saliva. Their presence in the saliva did not correspond to the variations of cyanosis, but the test was more often negative as the patient's condition began to improve. A bacillus which produced nitrites was grown from the saliva. He found the blood-serum also contained nitrites. Van den Bergh and Gutterink, after taking precautions to exclude nitrites in the air, did not find them in the serum, but found them in the corpuscles. They suggested that the nitrites might be formed by a specific micro-organism.

Dr. Wallis also found that the serum of these patients possessed the power of rapidly reducing oxyhaemoglobin. Observations were, therefore, made on these points.

\section{The Serum.}

Serum from the first specimen of blood withdrawn (November 2) gave a positive reaction to the metaphenylene-diamine test for nitrites. On the second occasion, when cyanosis was less definite, the serum did not show the presence of nitrites with the Griess-Ilosvay reagent (naphthylamine test).

In order to see if the serum of the patient's blood would reduce the oxyhaemoglobin of normal blood, a flat glass cell was made of such a thickness as to show the two oxybaemoglobin bands separately in a dilution of 1 in 20 of defibrinated normal blood. 0.5 c.c. of the diluted normal blood was put in the cell and 0.1 c.c. of the patient's serum (December 10) added and mixed gently. There was no change in the spectrum in half an hour at room temperature. Increasing additions of the patient's serum were then made to 0.5 c.c. of dilute normal blood, up to equal quantities of each, both at room temperature and al $40^{\circ} \mathrm{C}$., but no alteration of the spectrum resulted beyond the effect of the dilution. It is to be noted that cyanosis was not severe at this time; though the sulphaemoglobin band was present in the diluted blood, and could be seen through the lobe of the ear.

\section{The Urive.}

Daily analyses were made. Diacetic acid and acetone were present during the first week of admission, but later were usually absent. Albumin, sugar, blood, and indican were not found. The small amount of urine and of total nitrogen has been mentioned.

The proportion of ethereal to total sulpbates was found on five occasions to be very variable: $1: 5,1: 47,1: 22,1: 10,1: 3$. The second and fourth of these observations were made at the time of an attack.

During the attack of November 8 no nitrites were found in the urine; during that of November 30 a trace was present, and a faint trace on December 4 ; on the 6 th, 7 th, and 8 th none was found. On the last date there was a strong nitrite reaction in the saliva.

\section{Bacteriological Examination of Saliva.}

By Dr. Duncan, Lecturer on Bacteriology at the University of Aberdeen. The saliva gave a faint nitrite reaction. A similar reaction occurred in a small proportion of samples from normal individuals. The saliva was inoculated into nitrite-free gelatine, broth, and milk. The culture tubes were divided into two groups, one group being incubated under aerobic conditions, the second under anaerobic conditions. In each group tubes of every medium were incubated at room temperature, at $22^{\circ} \mathrm{C}$., and at $37^{\circ} \mathrm{C}$. Nearly all the tubes under all 
conditions gave a fairly copious mixed growth of bacteria, with a certain amount of gas-production in some cases. Tests were made at intervals up to a fortnight for the presence of nitrites, the Griess-Ilosvay reagent being employed in all cases. None of the tubes gave any nitrite reaction. Similar results were obtained with a second collection of saliva. The incubation of the saliva itself at $22^{\circ} \mathrm{C}$. and $37^{\circ} \mathrm{C}$. was also tried, but no nitrite reaction was obtained. Lastly, no nitroso-bacillus was found in the cultivations.

\section{Remarls on the Foregoing Tests.}

The observations made were to a large extent inspired by the interesting work of Dr. Mackenzie Wallis. It has long been known that sulphaemoglobin is formed when $\mathrm{H}_{2} \mathrm{~S}$ is passed through blood. But as $\mathrm{H}_{2} \mathrm{~S}$ is often present in the bowel, some explanation is needed as to why sulphaemoglobinaemia is rare. Van den Bergh thought that the slow constant development of $\mathrm{H}_{2} \mathrm{~S}$ in a condition of great intestinal delay was the cause of abnormal absorption. His view received support from the observations that his first patient, who suffered from a rectal constriction, recovered when the constriction was removed, and that his three other patients were all better so long as a daily action of the bowels was secured. Delay in the bowel, however, or even obstruction, is also much commoner than sulphaemoglobinaemia, suggesting the necessity of some additional factor.

Wood Clarke and Hurtley having shown that sulphaemoglobin can be formed from oxyhaemoglobin by strong reducing agents in the presence of the merest traces of sulphurotted hydrogen, Dr. Wallis, following up their work, suggested that a nitroso-bacillus, which he found in the saliva, formed, in addition to nitrites, a strong reducing substance which is easily absorbed into the blood-stream, a trace of sulphurotted hydrogen being presumably derived from the intestinal tract. He found the nitrite-producing bacillus in the saliva of the five cases bo examined. When growth of this bacillus occurred with utilization of amino acid a strong reducing substance appeared in addition to the nitrites. Further, in three cases in which it was tried, an addition of the patient's serum to a dilute solution of normal blood led to the replacement of the oxyhacmoglobin bands by the band of reduced hacmoglobin, with a corresponding change of colour of the solution, indicating the presence in the serum of these patients of a reducing substance more potent than could be explained by the presence of nitrites.

Dr. Wallis concludes that it is ' highly probable that this nitroso-bacillus had some definite relation to sulphaemoglobinaemia, but until more cases have been invostigated no definite pronouncement can be made'.

The idea that the blood of the patient contains a reducing substance, the presence of which in increased quantity causes the attacks of severe cyanosis, is an attractive one, and would also explain the good effect of the inhalation of oxygen. It may be remarked, however, that the observations hitherto recorded do not show that the reducing substance produced by the growth of the bacillus 
in vitro is the same as that found in the patient's serum. It would be of interest also to know whether the strong reducing power described is possessed by the plasma; and whether the serum or the plasma can reduce a solution of the patient's own blood; or if it must be assumed that the patient is in part immune from its full activity, as extensive reduction of oxyhaemogiobin to reduced haemoglobin in the arteries as well as in the capillaries would presumably be incompatible with life unless the lungs reoxygenated the blood; whereas this complaint is not a fatal one, and in our patient, even when cyanosis was severe, there was no dyspnoea. The circulating blood as seen in the lobe of the car appeared natural as regards the oxyhaemoglobin bands. If a reducing agent of such strength were present during life, it would have to be supposed that whilst it was active in aiding traces of $\mathrm{H}_{2} \mathrm{~S}$ to form sulphaemoglobin, it was prevented from reducing oxybaemoglobin to any great extent.

In this case Dr. Duncan failed to obtain a nitroso-bacillus from the saliva on two occasions, the technique foliowed being similar to that described by Dr. Wallis. Further, the serum of this patient was not found to have the power of reducing the oxylaemoglobin of a solution of normal blood. The sulphaemoglobin band was present in the blood when this test was made, but the cyanosis was milder than it had been. The reducing power of the serum from blood drawn during an attack of severe cyanosis with unconsciousness was not tested.

\section{Treatment.}

During the attacks of vomiting all food was either refused or vomited, and saline rectal infusions were given. Gastric and intestinal sedatives, such as bismuth, belladonna, morpbia, and chloretone, had little effect either on the sickness or pain. For sleeplessness, bromides, chloral, veronal, and Dial (Ciba) were used frequently.

Before the condition was recognized as one of sulphaemoglobinaemia, a purely milk diet, which van den Bergh found successful in cases of methaemoglobinaemia with diarrhoea, was tried for a few days with no benefit; it was much disliked by the patient.

Later, a mainly carbohydrate dict, as recommended by the experience of otbers, consisting of such foods as bread, semolina, macaroni, boiled rice, starchy breakfast foods, plain cake, and biscuits, was taken. At the same time treatment was directed to clearing and cleansing the bowel by purges, douches, and intestinal antisepties. Sulphate of magnesia in repeated doses and sodium sulphate were used. The bowel was also washed out daily with warm saline solution. Permanganate of potash in 1-grain pills, izal in 2-minim capsules, and beta-naphthol in 5 -grain cachets were tried in turn. Such measures appeared to cause some improvement, both of the cyanosis and the abdominal discomfort. The diet was, however, irksome, and towards the end of November eggs, chicken, milk, vegetables, and fruit were given. These additions were followed by an aggravation of symptoms; vomiting recurred, abdominal pain increased, and eyanosis returned. A return to the former diet was again followed by improvement. During the last attack of unconsciousness, in November, oxygen was given and appeared to lessen the cyanosis and shorten the attack. 


\section{Later History.}

After the patient returned to her home the carious teeth were removed. Dr. C. C. Cuthbert has kindly informed us that she is much better and has put on some weight. 'She still has her turns of cyanosis, but they are not so severe, and not nearly so frequent. Very often six weeks or two months will elapse between them.'

\section{Remarks on the Clinical Features.}

The twelve cases known to us are those of van den Bergh, 1; van den Bergh and Gutterink, 3 ; West and Wood Clarke, 1; A. E. Russell, 1; Essex Wynter, 1; Wood Clarke and Curts, 1 ; Haldin Davis, 1 ; Mackenzie Wallis, 2; and the present case. Dr. Wallis's paper also included a re-examination of the case previously described by Haldin Davis.

Cyanosis was, of course, present in the twelve cases. Constipation was present in ten of them, and probably in eleven. It is remarkable that the only two cases which are reported as recovered are van den Bergh's case, in which constipation was cured by an operation on a rectal stricture, and Dr. Wallis's case, under the care of Dr. Garrod, in which the bowels were not constipated as a rule. A detailed account of symptoms in van den Bergh's second, third, and fourth cases is not given, but taking the others, the symptoms complained of, in order of frequency, were: weakness, in six cases; headache, in five; fainting or unconsciousness, sleeplessness, in four ; vomiting, anaemia, dyspnoea, bruising, dental caries, scanty urine, neurosis, pyrexia, in two cases each.

The characteristic features of the complaint are the cyanosis, constipation, debility, and headache. These were all prominent in our case. In addition there were nausea, frequent attacks of vomiting, pains in various parts of the body, numbness or a feeling of paralysis, sleeplessness, faintness, palpitation, scanty urine, and neurotic or hysterical manifestations. Two operations have been performed on account of the abdominal symptoms, but no sufficient cause for the complaint has been found, and it is possible that the constant and sometimes spasmodic abdominal pain could be accounted for by adhesions and more or less arrest of the intestinal contents, such as was found by X-ray examination. The vomiting was, we think, partly a neurosis. It is difficult to state the duration of the illness. The patient had never been healthy, always constipated, for many years suffered abdominal symptoms suggesting delay in the bowel, and had been diagnosed as a case of intestinal toxaemia. Attacks of cyanosis, associated with a semi-comatose condition, first occurred a year before admission, and this time may be taken as indicating the onset of sulphaemoglobinaemia.

Observations on the saliva as described by Dr. Mackenzie Wallis did not in this case yield the rosults be obtained as to the presence of a nitroso-bacillus. It is possible that the good results which followed the use of a vaccine in one of his cases were due to the removal of carious teeth. Benefit appeared to follow the extraction of bad teeth in this patient. It must he remarked, however, that 
one patient (Dr. Ringrose's) in whose saliva Dr. Wallis found a nitroso-bacillus had no natural teeth.

The prosent case tends to confirm the view heretofore taken, namely, that the cause of the condition is to be sought in the alimentary canal, and that continued purgation offers at present the best means of treatment.

\section{Summary.}

1. A case of sulphaemoglobinaemia is described showing the usual features of cyanosis-constipation, debility, and headaches. The band of sulphaemoglobin was demonstrated in the blood and could be scen through the lobe of the ear.

2 . The patient complained of much abdominal pain, for which two operations had been performed without any sufficient cause for the pain having been found.

3. There was a large element of neurosis.

4. Improvement followed a diet consisting mainly of carbohydrate foods, the use of purges and antiseptic medicines, and the removal of carious teeth; but the patient is still the subject of the complaint.

5. Attempts to confirm the findings, described in some other cases, of a nitriteproducing bacillus in the saliva and of a substance in the blood-serum which strongly reduces oxyhaemoglobin solutions were unsuccessful in this patient.

For the chemical examinations and much of the spectroscopic work we are indebted to our colleague Mr. A. B. Weir, B.Sc. Professor Niven, of Aberdeen University, kindly placed a large spectroscope at our disposal for measuring the wave-lengths of the absorption bands. The bacteriological examinations wore conducted by Dr. George Duncan, of Marischal College, Aberdeen. We have made free use of clinical notes by $\mathrm{D} 1$. $D$. Pickering. To these gentlemen we wish to express thanks.

\section{REFERENCES,}

1. van den Bergh, Deutsch. Archiv f. klin, Med., 1905, 1xxxiii. 86 ,

2. van den Bergh u. Gutterink, Berl. klin. Woch, 1906, xliii. 7.

3. Gibson and Douglas, Lancet, Lond., 1906, ii. 72.

4. Wood Clarke and Hurtley, Journ. Physiol., Camb., 1907-8, xxxvi. 62.

5. Russell and Leathes, Lancet, Lond., 1907, i. 659; also Tians. Path. Soc, Lond., 1907, lviii. 177.

6. West and Wood Clarke, Med.-Chir. Trans., Lond., 1907, xc. 541.

7. Fusex Wynter, Proc. Roy. Soc. Med., Lond, (Clin. Sect.), 1907-8, i. 48, 197.

8. Wood Clarke, Med. Recird, New York, 1909, lxxvi. 143.

9. Wood Clarke and Curts, ibid., 1910, lxxviii. 987.

10. Haldin Davis, Lancet, Lond., 1912, ii, 1145.

11. Mackenzie Wallis, Quart. Journ. Med, Oxford, 1913-14, vii. 73. 